\title{
Surface Modifications for Improved Delivery and Function of Therapeutic Bacteria
}

\author{
Ava M. Vargason, Shruti Santhosh, and Aaron C. Anselmo*
}

Live therapeutic bacteria (LTBs) hold promise to treat microbiome-related diseases. However, few approaches to improve the colonization of LTBs in the gastrointestinal tract exist, despite colonization being a prerequisite for efficacy of many LTBs. Here, a modular platform to rapidly modify the surface of LTBs to enable receptor-specific interactions with target surfaces is reported. Inspired by bacterial adhesins that facilitate colonization, synthetic adhesins (SAs) are developed for LTBs in the form of antibodies conjugated to their surface. The SA platform is nontoxic, does not alter LTB growth kinetics, and can be used with any antibody or bacterial strain combination. By improving adhesion, SA-modified bacteria demonstrate enhanced in vitro pathogen exclusion from cell monolayers. In vivo kinetics of SA-modified LTBs is tracked in the feces and intestines of treated mice, demonstrating that SA-modified bacteria alter short-term intestinal transit and improve LTB colonization and pharmacokinetics. This platform enables rapid formation of an intestinal niche, leading to an increased maximum concentration and a $20 \%$ improvement in total LTB exposure. This work is the first application of traditional pharmacokinetic analysis to design and evaluate LTB drug delivery systems and provides a platform toward controlling adhesion, colonization, and efficacy of LTBs.

\section{Introduction}

The gastrointestinal (GI) microbiome plays essential roles in human health, including critical metabolic, immune and antivirulence functions. ${ }^{[1]}$ Due to this importance, live therapeutic bacteria (LTB), a class of commensal and nonpathogenic bacteria that can modulate the composition of the microbiome and mediate diseases related to it, are of increasing interest. ${ }^{[2]}$ Clinical evidence demonstrates that LTBs have the potential to treat Clostridium difficile infections, ${ }^{[3]}$ ulcerative colitis, ${ }^{[4]}$ cancer, ${ }^{[5]}$ and metabolic conditions (including phenylketonuria ${ }^{[6]}$ and hyperammonemia $\left.{ }^{[7]}\right)$. Despite differences in mechanisms of action, it is essential that the LTBs persist in the gut either permanently

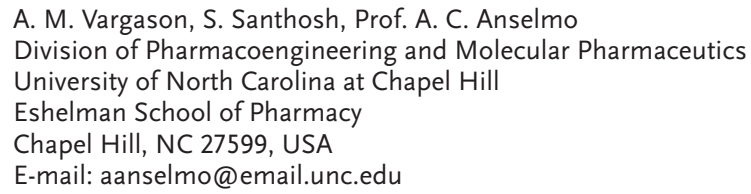

The ORCID identification number(s) for the author(s) of this article can be found under https://doi.org/10.1002/smll.202001705.

DOI: 10.1002/smll.202001705 (colonization) or for a sufficient transient period of time to provide efficacy. ${ }^{[8]}$ However, controlled persistence of LTBs in the gut remains a primary challenge to their clinical translation with few existing technologies to overcome limitations associated with LTB colonization, resulting in high recurring oral doses or invasive rectal administration. ${ }^{[9]}$ Some commensal and pathogenic bacteria address this challenge using surface proteins called adhesins that enable them to adhere to the intestinal lumen, facilitating persistence and colonization in the gut. ${ }^{[10]}$ By adhering to and colonizing the GI tract, commensal bacteria form a natural barrier to pathogen invasion, known as colonization resistance. ${ }^{[1 b, 11]}$ Therefore, the interactions between the microbial surface and the GI lumen are essential for persistence, colonization, and efficacy.

There is increasing interest in applying traditional drug delivery approaches to the microbiome field to improve LTB engraftment, persistence, and colonization. Previous work has applied encapsulation technologies to improve the colonization of LTBs by increasing their viability during gastric transit. ${ }^{[12]}$ However, encapsulation can block the LTB's interaction with the GI lumen and have downstream consequences on colonization. An alternative approach is to engineer the LTB surface to control its interaction with its site of interest. This has been immensely successful for particle-based drug carriers, where conjugation of adhesive ligands to the particle surface improves drug targeting, efficacy, and pharmacokinetics. ${ }^{[13]}$ Previous efforts to target LTBs and modify their surface have largely focused on cancer therapies, where LTBs have been used to naturally home to tumor sites and have been aided by mammalian cell coatings that shield their surface from the immune system. ${ }^{[14]}$ Surface modifications for pathogen exclusion or colonization have been limited to genetic engineering techniques that require tractable probiotic strains, high retention of plasmid DNA or precise genomic edits and show little modularity across LTB strains and adhesin targets. ${ }^{[12 \mathrm{~d}, 15]}$ As such, there is a need to develop modular, material-based platforms that control and enhance LTB interactions with their environment to improve colonization, while being compatible with diverse LTB species and GI targets.

In this manuscript, we describe a bio-inspired technology that rapidly modifies the LTB surface with synthetic adhesins, 
which mimic the functions of natural adhesins. By using biocompatible ester-amine chemistry to conjugate synthetic adhesins to the surface of LTBs, we show improved attachment to abiotic surfaces, monolayers of mammalian cells, and the mouse GI tract. This technology: i) is nontoxic to live bacteria, ii) can be applied to any synthetic adhesin target or LTB species or consortia, and iii) translates to enhanced in vitro and in vivo LTB performance due to improved colonization kinetics in the GI tract. Specifically, we s how that p rophylactic treatment of mammalian cells with modified LTBs significantly improves their colonization resistance, resulting in decreased pathogen attachment. Additionally, using fecal samples as a proxy for intestinal LTB concentration, we found that synthetic adhesins improve the in vivo pharmacokinetics of LTBs, including their rate of colonization, maximum concentration, and the total exposure over time. We further confirmed that $\mathrm{f}$ ecal $\mathrm{s}$ amples a re a $\mathrm{n}$ a ccurate $\mathrm{r}$ epresentation of intestinal LTB concentration and tracked viable LTB load in the intestinal tract and feces of mice to determine the effect of synthetic adhesins on both short-term intestinal LTB transit and longer-term niche formation. Altogether, our data demonstrates that the pharmacokinetic improvement provided by synthetic adhesins is a result of an initial increased abundance in the small intestine and cecum, leading to improved niche formation along the intestinal tract in the 3-days postadministration. This technology represents a rapid, tunable approach that can address colonization challenges by controlling specific interactions between the LTB a nd its a dhesion target.

\section{Results and Discussion}

\subsection{LTB Surface Modification}

To demonstrate the feasibility and modularity of chemically conjugating synthetic adhesins to the surface of live bacteria, we conjugated biotin to the surface of three bacterial species: Lactobacillus casei (LC), Escherichia coli (EC), and Bacillus coagulans (BC). Biotin is commonly used in bioconjugation reactions because of its strong and specific interaction with streptavidin, its designation as generally recognized as safe (GRAS), and its common use as an oral supplement. ${ }^{[16]}$ Biotin was conjugated to the surface of bacteria using N-hydroxysuccinimide ester (NHS) chemistry, which reacts with ubiquitous primary amines on bacterial surfaces (Figure 1a) to form amide bonds. The viability of each species was unaltered following biotinylation, demonstrating that NHS-ester chemistry is nontoxic to bacteria (Figure 1b). Biotinylation was confirmed a nd quantified us ing a flu orescent streptavidin probe that selectively bound to the surface of biotinylated bacteria (Figure 1c). Fluorescence from biotinbound streptavidin probes was quantified using a microplate reader, revealing that the three bacterial species demonstrate differences in the extent of s urface b iotinylation (Figure S 1, Supporting Information). These differences $m$ ay $b$ e a ttributed to varying primary amine densities, surface charges, and total surface area between the three bacterial species. We further confirmed the m odularity o $\mathrm{ft}$ his a pproach $\mathrm{b} y$ applying surface modifications to the commercially available probiotic consortia Visbiome. Following biotinylation, streptavidin was able to bind bacterial species in the Visbiome consortium with high specificity compared to an unmodified control (Figure S2, Supporting Information). Scanning electron microscopy (SEM) revealed no signs of morphological differences between the unmodified and biotinylated bacteria (Figure 1d), a standard indicator of bacterial damage to the cell wall. ${ }^{[17]}$ Finally, we demonstrated that the growth behavior for all strains was not affected by biotinylation (Figure 1e).

\subsection{LTB Attachment}

To determine whether biotinylation of bacteria significantly alters their attachment to surfaces, we quantified bacterial attachment to an abiotic streptavidin-coated well-plate and to monolayers of mammalian cells. For these studies, an engineered strain of EC DH5 $\alpha$ expressing GFP was used to quantify attachment of bacteria. ${ }^{[18]}$ Both biotinylated and unmodified bacteria were incubated on a streptavidin-coated plate for $1 \mathrm{~h}$ at varying concentrations. Following washes, biotinylated bacteria attached at significantly higher quantities than unmodified bacteria for all concentrations tested (Figure 2a,b). The attachment of biotinylated bacteria showed a strong, dose-dependent and linear relationship (Figure $2 \mathrm{~b}$ ).

In the GI tract, probiotic bacteria must adhere to human tissue, mucus or cells to prevent mechanical clearance due to peristalsis and mucus turnover. ${ }^{[10]}$ To enhance the adherence of biotherapeutics to mammalian cells, we attached monoclonal antibodies to the surface of biotinylated bacteria by conjugating streptavidin groups to the constant region of the antibody (Figure 2c). Monoclonal antibodies are highly effective at targeting therapeutics to specific cells and cellular targets. ${ }^{[13 c, 19]}$ We confirmed antibody conjugation using a native protein gel (Figure S3, Supporting Information) and attachment of the conjugate to the bacterial surface using fluorescence and zeta potential (Figure S4, Supporting Information), which has previously been used to assess bacterial surface charge and confirm surface modifications. ${ }^{[12 a, 20]}$ To target the carcinoma cell-line Caco-2, which is frequently used as a model of the intestinal barrier, we conjugated streptavidin to a monoclonal antibody against Intracellular Adhesion Molecule (aICAM-1), to specifically bind to surface-expressed ICAM-1 receptors on Caco-2 cells. ${ }^{[21]}$ ICAM-targeted and unmodified bacteria were incubated with Caco-2 cells for $1 \mathrm{~h}$ before thoroughly washing to remove unbound bacteria. As expected, the ICAM-targeted bacteria attached in significantly higher amounts than the unmodified control (Figure 2d,e). To confirm that EC attachment was due to successful presentation of streptavidin-functionalized aICAM-1 on biotin-modified bacteria, a panel of controls were analyzed. Biotinylated and unmodified bacteria were pre-incubated with aICAM-1 or the aICAM-streptavidin conjugate to evaluate whether surface conjugation, as opposed to passive adsorption, was required to provide targeted functionality. Controls demonstrate that surface functionalization with biotin and subsequent attachment of the aICAM-streptavidin conjugate is required for sufficient antibody display and improved bacterial attachment to Caco-2 cells (Figure 2d). 
A
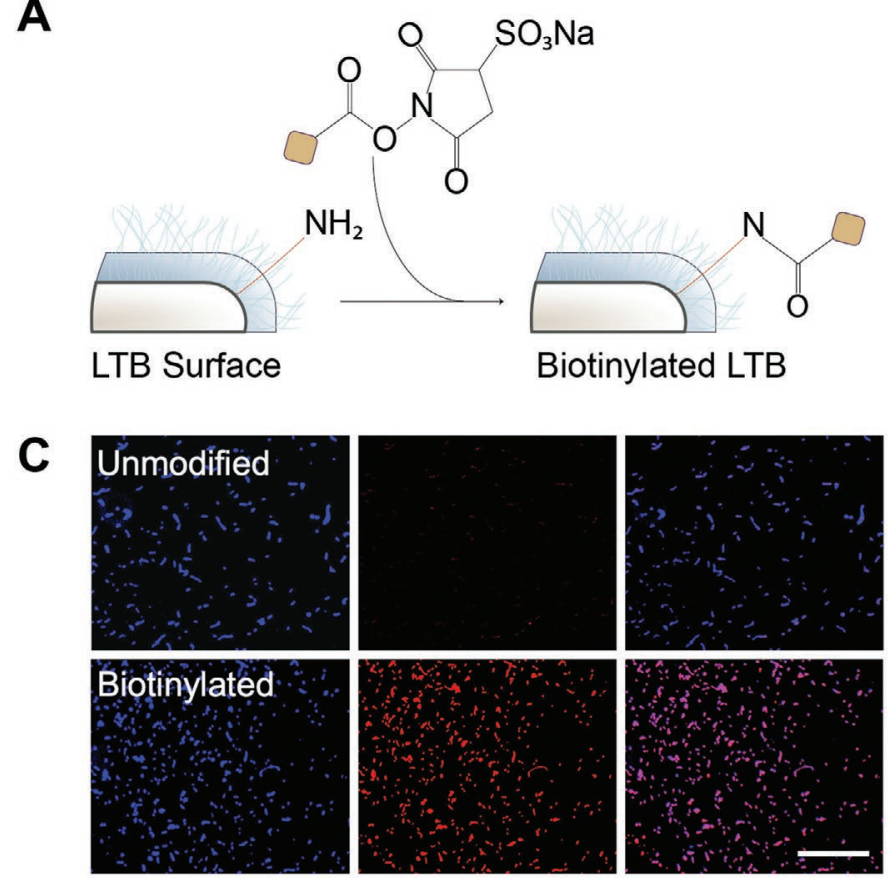

LC
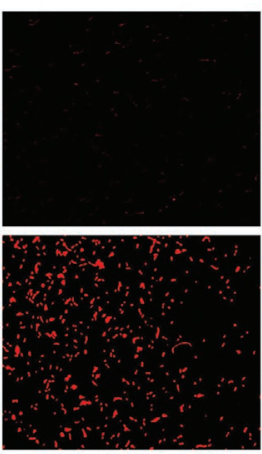

Streptavidin
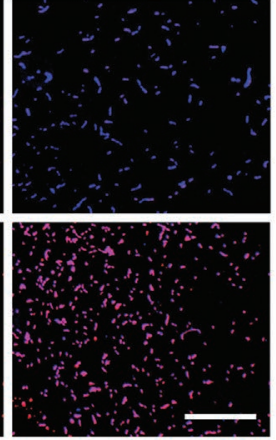

Overlay
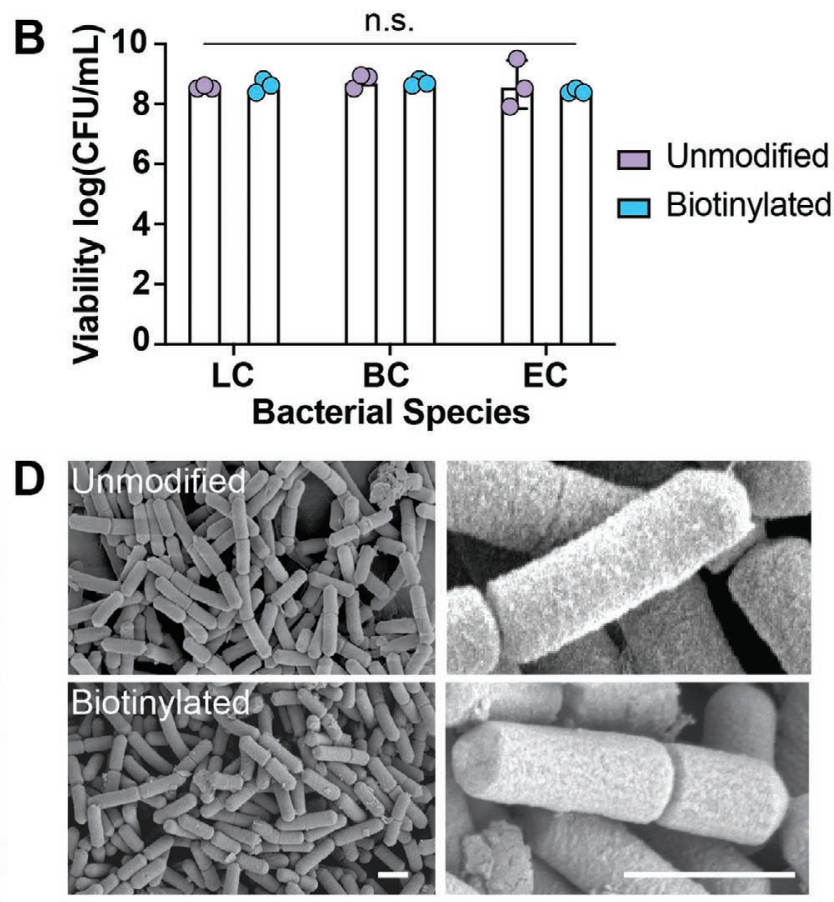

E

E. coli

L. casei

B. coagulans
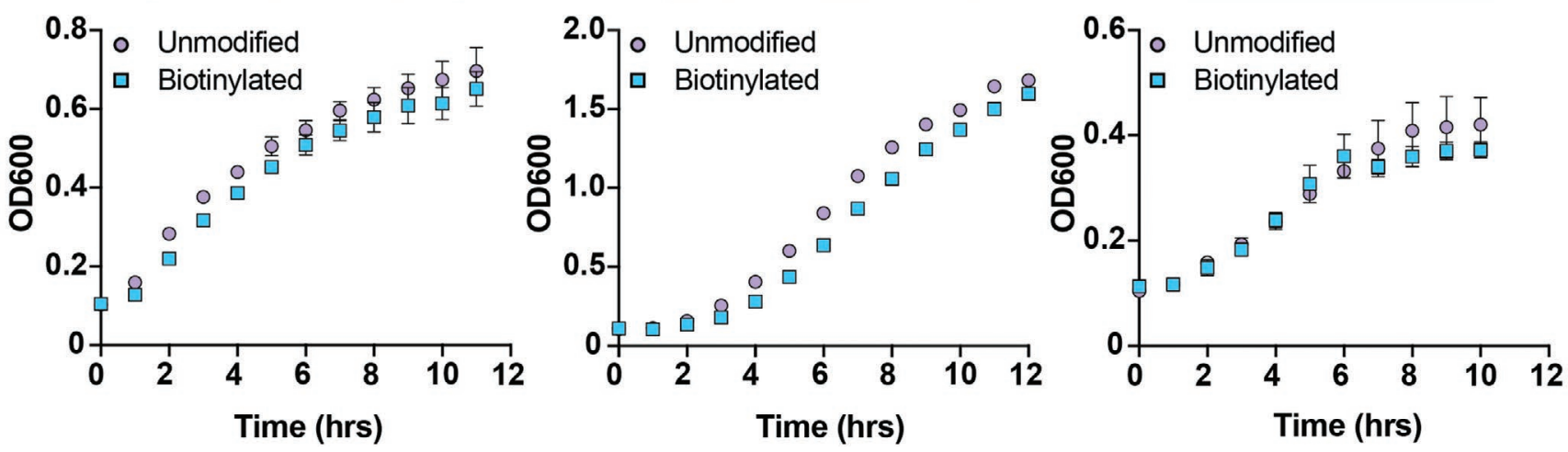

Figure 1. Modular biotinylation of probiotic bacteria. A) Schematic of $\mathrm{N}$-hydroxysulfosuccinimide ester chemistry for bioconjugation of biotin to primary amines on the bacteria surface. B) Viability of all three species prior to and following biotinylation reaction (error represents standard deviation, $n=3$, significance assessed using multiple unpaired Student's t-tests). C) Epi-fluorescence images of unmodified (top) and biotinylated (bottom) LC following incubation with Alexa Fluor Streptavidin Conjugate. D) SEM images of unmodified (top) and biotinylated (bottom) LC. E) Growth studies of biotinylated and unmodified EC, LC and BC (error represents standard deviation, $n=3$ ). Epi-fluorescence scale bar $=50 \mu \mathrm{m}$. SEM scale bars $=1 \mu \mathrm{m}$.

\subsection{Colonization Resistance Assay}

A known beneficial and microbiome-regulatory function of commensal bacteria is the prevention of pathogen attachment and colonization in the GI tract. ${ }^{[11]}$ Motivated by this natural function, fecal microbiota transplants (FMTs), ${ }^{[3 a]}$ engineered LTBs $^{[15 b]}$ and probiotic formulations ${ }^{[22]}$ have been developed to exclude and compete with pathogens. ${ }^{[23]}$ This anti-adhesive approach holds promise as both a treatment for pathogen infections, as well as a prophylactic to prevent pathogens from initially colonizing. We tested whether our system could be used as an anti-adhesion therapy by preventing a model pathogen from attaching to epithelial cells (Figure 3a). For this study, we used the common dairy probiotic species LC, which has anti-inflammatory and anti-virulence properties. ${ }^{[24]}$ Furthermore, Lactobacillus species have been shown to mediate pathogen attachment by forming a steric barrier on mammalian cells or the mucosal lining, which we hypothesized could be enhanced by the addition of synthetic adhesins targeted to Caco- 2 cells. ${ }^{[25]}$ By targeting LC to ICAM-1, LC adherence to Caco-2 cells is significantly increased compared to an unmodified control (Figure $3 \mathrm{~b}$ ), determined by plating and enumerating viable CFUs following the removal of the Caco-2 monolayer.

We next analyzed whether prophylactic treatment of Caco-2 cells with LC can reduce subsequent attachment of a bacterial 

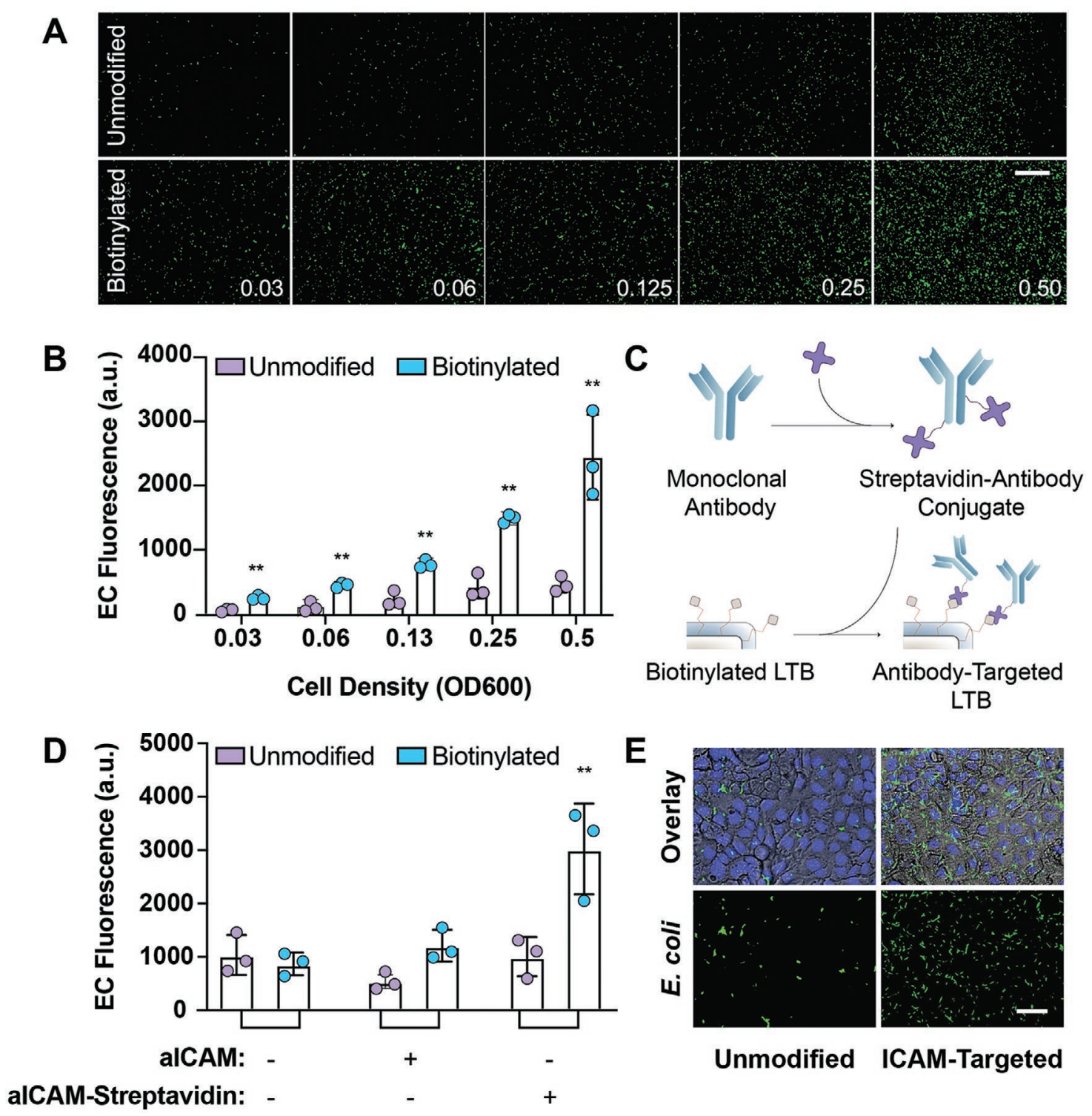

Figure 2. Attachment of biotinylated bacteria to abiotic and biotic surfaces. GFP-expressing EC were used to quantify attachment to surfaces. A) Representative images of unmodified (top) and biotinylated (bottom) bacteria at increasing optical densities (OD600) and B) quantification of the concentration-dependent attachment of unmodified and biotinylated bacteria to a streptavidin-coated well-plate (error represents standard deviation, $n=3$, significance assessed using multiple unpaired Student's t-tests, $* * x<0.01)$. C) Streptavidin conjugation to the constant region of IgG antibodies enables antibody attachment to the surface of biotinylated bacteria. D) Attachment of unmodified and biotinylated bacteria to a monolayer of Caco-2 cells after no incubation or incubation with an anti-ICAM antibody (aICAM) or anti-ICAM-streptavidin conjugate (alCAM-streptavidin) (error represents standard deviation, $n=3$, significance assessed using two-way ANOVA with Sidak's multiple comparisons, $* * x<0.01)$. E) Representative images of bacteria attached to Caco-2 monolayers. Scale bars $=(A) 130 \mu \mathrm{m}$ and $(\mathrm{E}) 65 \mu \mathrm{m}$.

pathogen. Common pathogenic bacterial species show significant toxicity toward mammalian cells, leading to compromised integrity of the Caco-2 monolayer. For our in vitro model, we found that this toxicity limited the use of standard quantitative analysis methods due to the compromised monolayer, leading to high rates of pathogen attachment to the polystyrene well plate (Figure S5a, Supporting Information). To maintain Caco-2 monolayer integrity and accurately quantify bacterial attachment to Caco-2 cells, we instead selected a GFP-expressing EC DH5 $\alpha$ strain as the model pathogen. Caco-2 cells were treated with either unmodified or ICAM-targeted LC for $1 \mathrm{~h}$. After washing the cells to remove unbound LC, Caco- 2 cells were challenged for $1 \mathrm{~h}$ with either an equal (1:1) or 10-fold higher (10:1) ratio of pathogen to probiotic (Figure 3c). ICAM-targeted LC was 3-fold more effective than the unmodified control in preventing EC attachment to Caco-2 cells (Figure 3c; Figure S6, Supporting Information). Interestingly, the efficacy of ICAM-targeted LC was independent of the pathogen:probiotic ratio, highlighting how a small population of targeted probiotics can be used to limit 


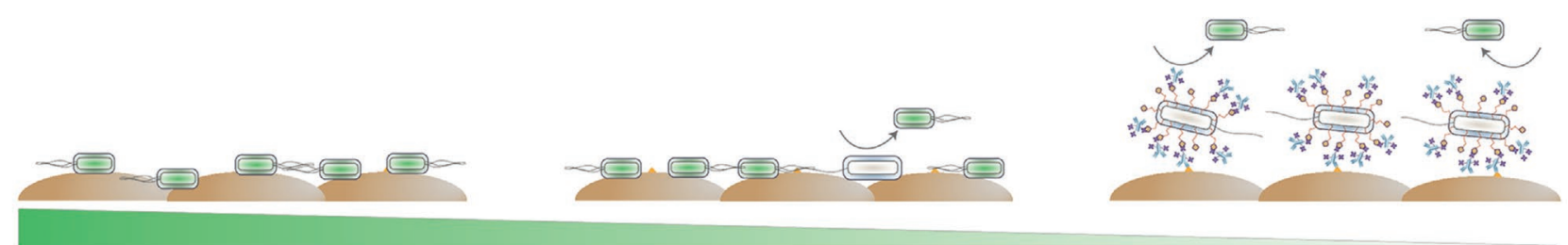

Decreasing E. coli attachment
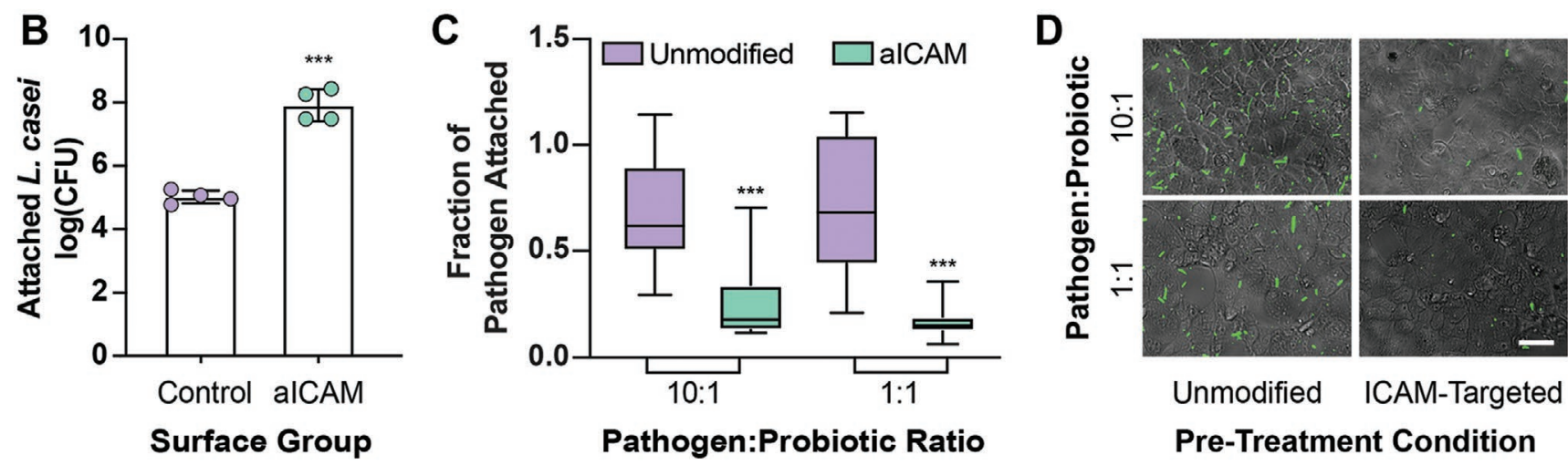

Figure 3. Synthetic adhesins improve pathogen exclusion from a monolayer of Caco-2 cells. A) Escherichia coli (EC) attachment to Caco-2 cells was quantified under three conditions: no probiotic pre-treatment (left), pre-treatment with unmodified Lactobacillus casei (LC) (middle) and pre-treatment with ICAM-targeted LC (right). B) CFU of attached unmodified and ICAM-targeted (aICAM) LC to Caco-2 monolayers were quantified via plating following $1 \mathrm{~h}$ of incubation (error represents standard deviation, $n=4$, significance assessed using unpaired Student's t-test, **** $p<0.001$ ). C) EC attachment following pre-incubation with unmodified or ICAM-targeted (aICAM) LC for $1 \mathrm{~h}$, followed by a $1 \mathrm{~h}$ challenge with EC. Results are normalized to the amount of EC attached without pre-incubation (error represents standard deviation, $n>15$ with at least 5 images per well and 3 wells per conditions, significance assessed using two-way ANOVA with Sidak's multiple comparisons, $*$ *⿰火火 $p<0.001)$. D) Representative images following challenge with GFP-expressing EC. Scale bar $=65 \mu \mathrm{m}$.

attachment of a pathogen, even when the pathogen is present at an order of magnitude higher concentration. Representative images demonstrate the reduction in EC attachment following treatment with ICAM-targeted LC (Figure 3d). The dramatic reduction in EC attachment to live Caco- 2 cells demonstrates that synthetic adhesins can be used to create a barrier against pathogen attachment by granting the probiotic an adherence advantage.

To investigate the benefit of targeting a general receptor on Caco-2 cells during a competitive challenge model, we incubated LC and EC simultaneously on Caco-2 cells. Following washes to remove unbound bacteria, we found that surface modification does not significantly affect the attachment of EC compared to unmodified LC (Figure S5b,c, Supporting Information). We believe that this is because our system relies on physically excluding pathogens after LTB binding, as opposed to directly competing with the pathogen for specific adhesin receptors. As such, targeting enhances the ability for LC to form a physical steric barrier that improves pathogen exclusion only in a prophylactic model. Modification of the LC surface with antibodies directed toward EC binding sites would likely provide a direct competitive advantage to $\mathrm{LC}$, as previous reports of genetically engineered probiotics that directly compete with pathogen binding can reduce and displace bound pathogen. ${ }^{[15 \mathrm{~b}]}$

\subsection{In Vivo Evaluation}

\subsubsection{Pharmacokinetics of $E c N$}

Due the importance of surface adhesins in the colonization of biotherapeutics in the GI tract, we next investigated the effect of synthetic surface modifications on in vivo colonization. E. coli Nissle 1917 (EcN), a probiotic with extensive clinical and preclinical data that naturally colonizes the GI tract, ${ }^{[26]}$ was used for in vivo studies. ${ }^{[27]}$ To enhance the adherence of EcN to the GI tract, anti-MUC2 antibodies (aMUC2) were attached to the surface as previously described. To reduce the cost of the platform and improve its potential for translation, we selected a polyclonal antibody for in vivo studies in contrast to the monoclonal anti-ICAM-1 antibodies used for in vitro studies. MUC2 is an essential component of intestinal mucus, a common adhesin target for bacteria, and a mediator of host-bacterial interactions at the mucosal interface, making it a ubiquitous and bio-inspired choice for a synthetic adhesin (SA). ${ }^{[28]}$ Prior to administration of $\mathrm{EcN}$, mice were pre-treated with streptomycin for 24-h, followed by an 18-h washout period of the antibiotic. Antibiotic pre-treatment is routinely used to enable LTB colonization in clinical settings, including for FMTs and LTB consortia. ${ }^{29]}$ Streptomycin specifically opens a niche for EcN colonization 

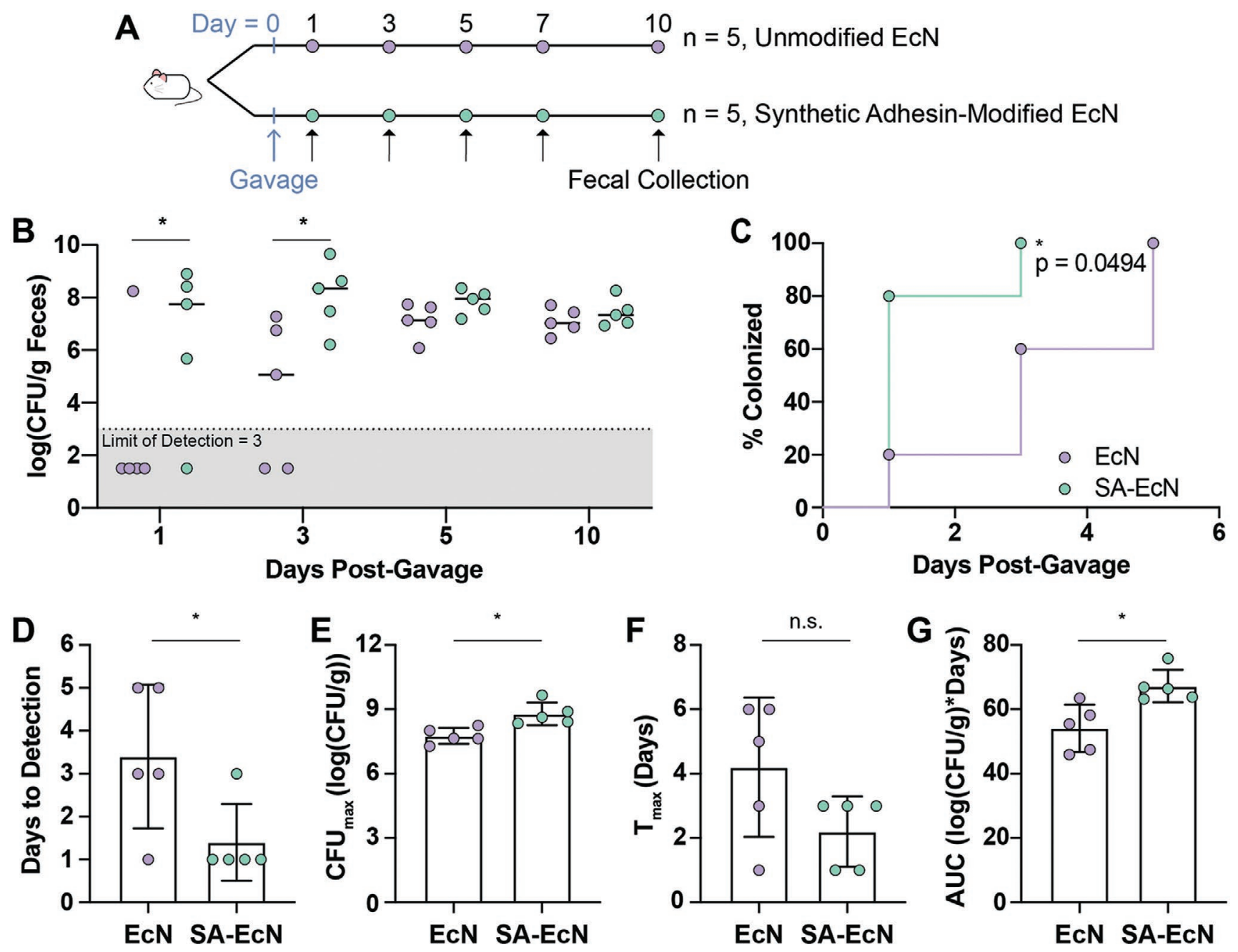

Figure 4. Pharmacokinetics of EcN colonization in BALB/c mice. A) Eight-week old female BALB/c mice were treated with streptomycin for 24 h, followed by an 18-h wash-out period. Mice were treated with unmodified or aMUC2 synthetic adhesin-modified (SA-EcN) EcN via oral gavage and fecal pellets were collected at indicated timepoints. B) Viable colony forming units (CFU) of EcN in feces were determined by homogenizing and plating fecal pellets at indicated timepoints for unmodified (purple) and synthetic adhesin (green) EcN (bars represent median, $n=5$, significance assessed using two-way ANOVA with Sidak's multiple comparisons, $* p<0.05$ ). C) Kinetics of colonization, defined as detectable EcN in feces ( $n=5$, significance assessed using Log-rank Mantel-Cox test, $* p<0.05$ ). D) Time to colonization for each mouse, defined as detectable EcN in feces (error represents standard deviation, $n=5$, significance assessed using unpaired Student's t-test). (E-G) Pharmacokinetics of EcN colonization in the murine GI tract, including E) maximum detected CFU in each animal, F) the time $\mathrm{CFU}_{\max }$ occurred in each animal and G) the area under the $\log \left(\mathrm{CFU} \mathrm{g}^{-1}\right)$-time $\mathrm{curve}$ for each animal (error represents standard deviation, $n=5$, significance assessed using unpaired Student's t-tests, * $p<0.05$, n.s. $=$ not significant).

by selectively removing facultative anaerobes, leaving the abundance and diversity of remaining anaerobes intact. ${ }^{[30]}$ In our model, we found that EcN fails to colonize mice in the absence of either antibiotic treatment or a wash-out period (Figure S7, Supporting Information). Unmodified and aMUC2 synthetic adhesin-modified (SA-EcN) EcN were delivered to mice via oral gavage and colonization was tracked over a period of 10 days (Figure 4a). Fecal pellets were used to quantify the intestinal EcN concentration, as fecal bacterial concentration has previously been used as a proxy for bacterial load in the intestines. ${ }^{[27,31]}$ Mice treated with SA-EcN had significantly higher bacteria in their feces on days 1 and 3 following gavage (Figure 4b) and both groups stabilized to approximately $10^{7} \mathrm{CFU} \mathrm{g}^{-1}$ feces by day 5. Defining colonization as the presence of detectable bacteria in the feces, we analyzed the length of time required for all mice in a group to become colonized (Figure 4c,d). Synthetic adhesins significantly reduced the time required to reach $100 \%$ colonization, with all mice in the SA-EcN treatment group having detectable $\mathrm{EcN}$ in their feces by day 3 .

To understand the effects of earlier colonization via synthetic adhesins on microbe-host interactions, we calculated pharmacokinetic parameters (Figure $4 \mathrm{e}-\mathrm{g}$ ) that are traditionally used to understand the absorption and elimination of a therapeutic. Previous work for live biotherapeutics has used pharmacokinetics to describe LTB colonization, ${ }^{[32]}$ or the effect that LTBs have on diagnostic read-outs and co-administered therapeutics. ${ }^{[33]}$ However, to our knowledge, no previous work has applied traditional pharmacokinetics to describe the benefits of 
a rationally designed delivery system for LTBs. Our results show that SA-EcN reached a significantly higher viable concentration $\left(\mathrm{CFU}_{\max }\right)$ than unmodified EcN (Figure 4e). Additionally, the time at which the $\mathrm{CFU}_{\max }$ occurs $\left(t_{\max }\right)$ is lower for SA-EcN (Figure 4f). Therefore, synthetic adhesins enable EcN to rapidly reach a high concentration in the GI tract. To determine the long-term consequences of the $t_{\max }$ and $C_{\max }$, we calculated the area under the curve (AUC) for both SA-EcN and the unmodified control. The AUC is the integral for the plot of EcN concentration in feces versus time (Figure $4 \mathrm{~b}$ ) and is a measure of the total exposure to a therapeutic. The AUC of SA-EcN is significantly higher than the unmodified control (Figure 4g). Therefore, even though synthetic adhesins do not lead to a long-term increase in colonization, their advantages at early timepoints increase an animal's total exposure to EcN by $20 \%$. For biotherapeutics that secrete small molecules or biologics, this would lead to a direct increase in the patient's exposure to their bioactive compounds.

The effect of synthetic adhesins is likely transient for two reasons: i) surface modifications dilute as bacteria proliferate in vivo and ii) mice are coprophagic. This system relies on chemical conjugation to the surface of bacteria, which will lead to dilution of the conjugated targeting ligands on the LTB surface as they grow. Therefore, as the bacteria grow in vivo, they lose their synthetic adhesins and, subsequently, their ability to specifically adhere to their synthetic adhesin's target. While the dilution of surface modifications on the LTB surface may be a limitation of the platform, it also represents an advantage compared to permanent alterations of the LTBs that may introduce safety concerns of administering genetically engineered bacteria or can interfere with the natural mechanism of action for the LTB. Furthermore, our in vivo data collectively demonstrate that the early advantages provided by antibody targeting of LTBs is sufficient to establish an intestinal niche, enabling them to proliferate in the GI tract and withstand clearance mechanisms such as peristalsis and mucosal clearance. In addition to the dilution of targeting ligands, the mice are not individually housed and therefore will ingest feces throughout the study, re-inoculating their intestinal tract with shed EcN. These two processes will saturate and stabilize the amount of EcN in the intestinal tract, as shown starting at day 5 (Figure $4 \mathrm{~b}$ ). Because coprophagy is unique to rodents, the benefits of synthetic adhesins may be understated by this data.

\subsubsection{Intestinal Abundance of $E c N$}

To investigate the effect of synthetic adhesins on the short-term transit of EcN in the intestinal tract, mice were gavaged with either SA-EcN or an unmodified control and sacrificed 1- and 4-h later (Figure 5a). To confer bioluminescence and image EcN on an In Vivo Imaging System (IVIS), a strain bearing no native plasmids was transformed with the pGEN-luxCDABE plasmid. ${ }^{[34]}$ The intestinal tracts were harvested and imaged using IVIS to visualize distribution of the bacteria along the GI tract (Figure S8, Supporting Information), which showed EcN in the small intestine at $1-\mathrm{h}$ postgavage and all segments of the GI tract by 4-h postgavage. The small intestine, cecum and colon were homogenized and plated to determine the viable abundance of $\mathrm{EcN}$ in each organ (Figure 5b). The plating data proved to be a more sensitive method for detecting and quantifying $\mathrm{EcN}$ in the intestinal tract, revealing that mice treated with SA-EcN have a significantly higher abundance of EcN in their cecum at 1-h, indicating faster transit than unmodified EcN. By $4 \mathrm{~h}$ postgavage, mice treated with SA-EcN have significantly higher viable bacteria in their small intestines and ceca. Synthetic adhesins therefore alter the transit of EcN in the GI tract, enabling a population of SA-EcN to reach the cecum faster (Figure 5b, left), while remaining $\mathrm{EcN}$ have an increased residence time in the small intestine. Additionally, SA-EcN appear to persist in the cecum at a higher abundance than the unmodified control (Figure 5b, right). This strongly supports the colonization data by highlighting that modification with synthetic adhesins results in both faster appearance and higher viable amounts of $\mathrm{EcN}$ in the feces of treated mice.

To confirm that the increased abundance of EcN in the feces of SA-EcN treated mice at early timepoints (Figure 4b) is an indicator of improved intestinal colonization rather than rapid transit and clearance, intestinal colonization was assessed as described above at 24 - and 72 -h postgavage. At $24-\mathrm{h}, 60 \%$ of the SA-EcN mice were colonized in all segments of their intestinal tract, including the small intestine, cecum, and colon (Figure 5c, left). While three control mice had detectable EcN in their small intestine, none were colonized throughout their GI tract and by 72-h, only SA-EcN treated mice had viable EcN in the intestinal tract (Figure 5c, right). The intestinal tracts were additionally imaged using IVIS, which showed $\mathrm{EcN}$ in the intestinal tract of mice in both groups at 24-h, but only SA-EcN treated mice by 72-h (Figure S9, Supporting Information). Importantly, none of the mice in the control group had viable EcN in their feces at either 24- or 72-h postgavage (Figure S10a,b, Supporting Information). To determine the relationship between fecal and intestinal samples, we next correlated the abundance of EcN in the feces and intestinal tracts in two groups of mice: those with detectable EcN in their feces (colonized) and those without (noncolonized) (Figure 5d). We found that colonized mice had comparable levels of $\mathrm{EcN}$ in their feces and intestinal tracts (Figure 5d, right), while noncolonized mice showed lower or no viable $\mathrm{EcN}$ in their intestinal tracts (Figure $5 \mathrm{~d}$, left). From this data, we conclude that the presence of EcN in feces is indeed indicative of intestinal $\mathrm{EcN}$ colonization.

Taken together, our data demonstrates that in all cases where fecal counts are detectable, the intestinal tract is colonized with a comparable level of $\mathrm{EcN}$ (Figure $5 \mathrm{~d}$ ). From this, it is clear that treatment with SA-EcN leads to higher abundance in the small intestine and cecum immediately following administration (Figure 5b), enabling improved intestinal and fecal colonization in the first three days (Figures $4 \mathrm{~b}$ and $5 \mathrm{c}$ ). Therefore, we hypothesize that synthetic adhesins improve the ability of EcN to rapidly form an intestinal niche that acts as a stable depot to sustain shedding of excess EcN into the feces. This agrees with literature on probiotic and commensal species, where the fecal microbiome is frequently used as a proxy for the intestinal environment, ${ }^{[27,31]}$ as well as known mechanisms of pathogen colonization, where formation of an intestinal niche supports a sustained intestinal population that is responsible for fecal shedding. ${ }^{[35]}$ Finally, this hypothesis is further supported by the fact that all mice in our long-term colonization study were 
A

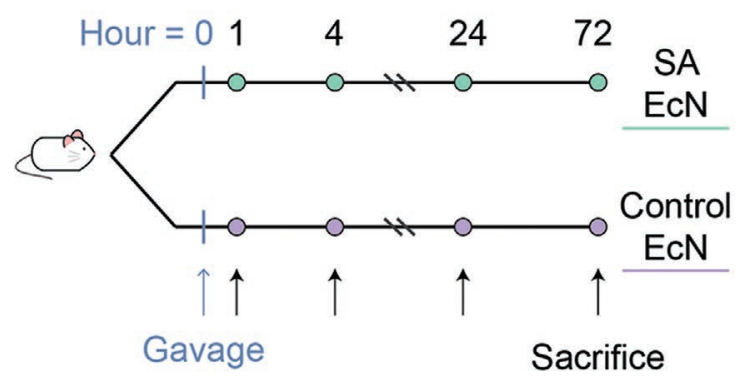

$B$ ธิ 1.5

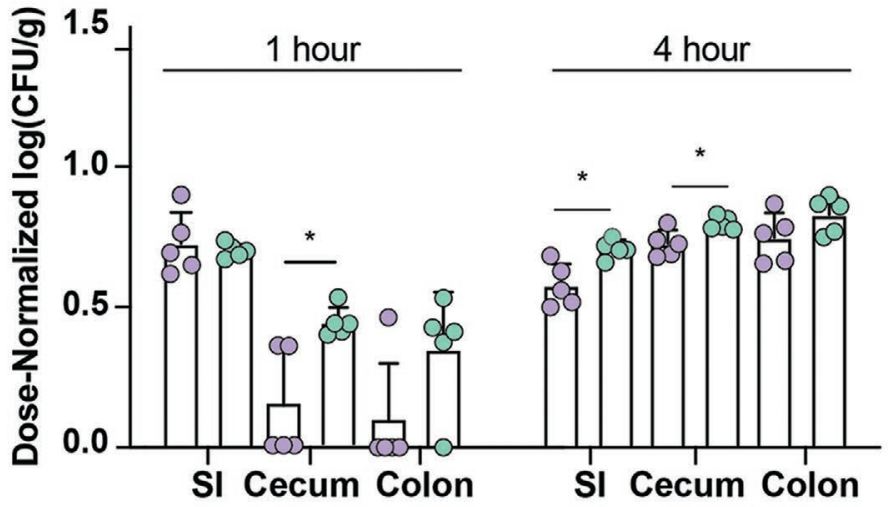

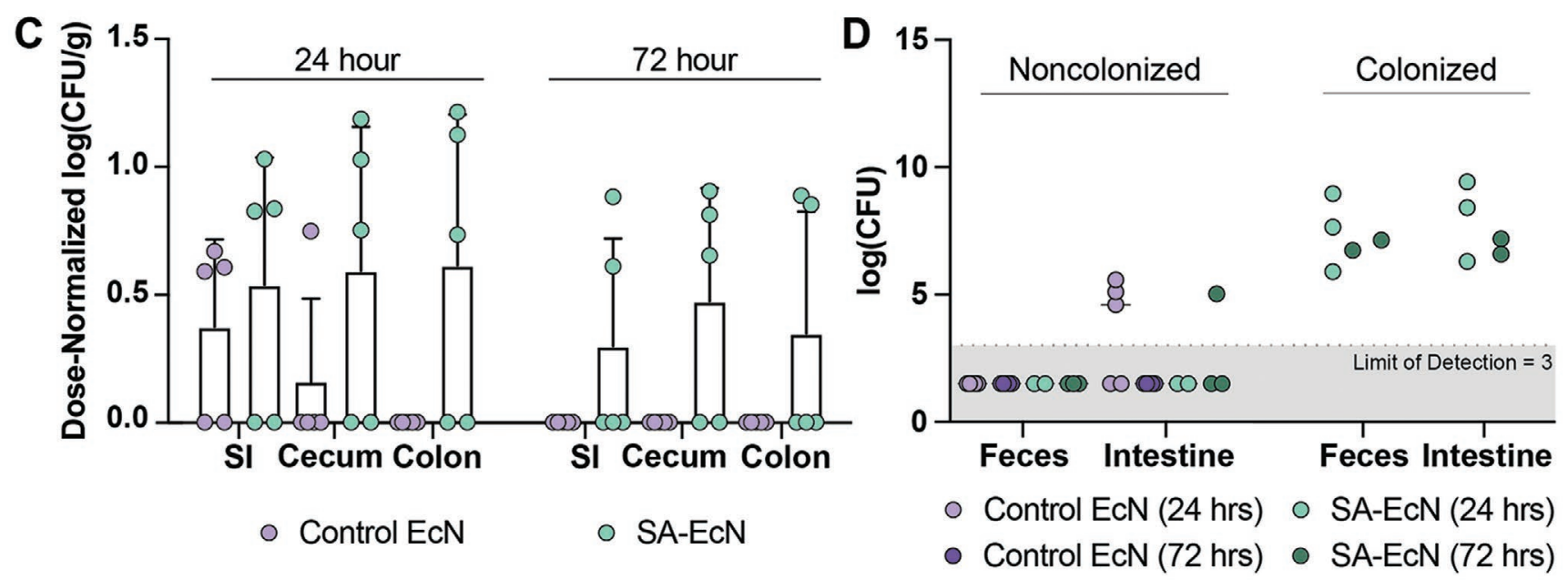

Figure 5. Distribution and abundance of EcN in the intestinal tract postgavage. A) Eight-week old female BALB/c mice were dosed with unmodified or aMUC2 synthetic adhesin-modified (SA-EcN) EcN via oral gavage and sacrificed 1-, 4-, 24-, or 72-h later. Intestines were harvested and EcN abundance was evaluated by plating. B) Abundance of EcN in the small intestine (SI), cecum, and colon of mice 1- (left) and 4-h (right) postgavage (error represents standard deviation, $n=5$, significance assessed using multiple unpaired Student's t-tests, * $p<0.05)$. C) Abundance of EcN in SI, cecum, and colon of mice 24- (left) and 72-h (right) postgavage (error represents standard deviation, $n=5$, significance assessed using multiple unpaired Student's t-tests, no significant differences between groups). D) Concentration of EcN in feces and entire intestinal tract from mice with no viable counts in their feces (noncolonized, left) and viable counts in their feces (colonized, right) (bars represent median). Abundance is dose-normalized to account for variations [Dose-Normalized $\log \left(\right.$ CFU g $\left.{ }^{-1}\right)=\log \left(C F U g^{-1}\right.$ Detected in Organ)/log(Dose Administered)] in (B,C).

stably colonized with EcN at least a month following treatment (Figure S10c, Supporting Information), suggesting an equilibrium between EcN growth and fecal shedding during this time.

\section{Conclusion}

Overall, this work demonstrates a rapid and modular platform that can be used with any given bacteria and antibody combination to modify the bacterial surface, including over-thecounter probiotics, beneficial consortia, and LTBs used in the clinic. We have shown that surface modification improves LTB adhesion, enhancing the ability to exclude pathogenic bacteria in vitro, even in the presence of a 10-fold higher pathogen burden. Additionally, this manuscript presents a new perspective on LTB pharmacokinetic analysis, providing a framework for designing and evaluating engineered drug delivery systems for LTBs. Using this analysis, we demonstrated that synthetic adhesins enable an early colonization advantage that supports an intestinal LTB depot, leading to an increase in their maximum concentration and the total exposure to the biotherapeutic over time without impeding subsequent LTB growth in, or interaction with, the GI tract. For LTBs engineered to secrete biotherapeutics or for those that are active for only a short window following administration, such as Synlogic's Phase I/II candidate SYNB1618, ${ }^{[36]}$ this early advantage in colonization and proliferation in the intestinal tract will directly correlate with improved patient exposure to the biotherapeutic and efficacy of the LTB.

Future work will be focused on addressing the potential limitations of our targeted LTB system. These limitations include the requirement of antibiotics to achieve LTB colonization, which can contribute to antibiotic resistance or exacerbate disease symptoms, and the potential for altered LTB distribution and tissue localization in the GI tract, posing risks for small intestine bacterial overgrowth (SIBO). ${ }^{[37]}$ The translation of targeted LTBs will also face clinical hurdles such as large-scale labeling and storage of LTBs, requiring careful consideration 
of the cost and in vivo stability of the LTB targeting ligand. Toward potential translational applications, we designed our LTB-targeting system to be compatible with postmanufactured, postpackaged, and commercially available probiotic species (Figure S2, Supporting Information). Additionally, the functionalization chemistry relies on amine groups that are present on many targeting ligands, making the system amenable to a range of targeting moieties such as synthetic peptides, mucoadherent polymers, or aptamers as an alternative to antibodies which may show limited in vivo stability. Collectively, this manuscript presents a modular approach toward improving the efficacy and colonization of LTBs in the GI tract and provides a proof-of-concept platform that can be applied in future work for the treatment of microbiome-related diseases.

\section{Experimental Section}

Cell Lines and Culture: Caco-2 (ATCC HTB 37) cells were purchased from the University of North Carolina at Chapel Hill Tissue Culture Facility. Caco- 2 cells were cultured in DMEM media supplemented with $1 \%$ penicillin-streptomycin and $10 \%$ Fetal Bovine Serum (FBS). Lactobacillus casei (ATCC 393) and Bacillus coagulans (ATCC 7050) were purchased from ATCC. Escherichia coli $\mathrm{DH} 5 \alpha$ was purchased transformed with a pBS-IdhGFP plasmid, a gift from Michela Lizier (Addgene plasmid \#27 170; http://n2t.net/addgene:27170; RRID:Addgene_27 170). ${ }^{18,34]}$ Escherichia coli Nissle 1917 was a gift from Nathan Crook and was transformed with the pGENlux-CDABE plasmid, a gift from Harry Mobley (Addgene plasmid \#44 918; http://n2t.net/addgene:44918; RRID:Addgene_44 918). ${ }^{[27]}$ All bacterial cultures were inoculated from glycerol stocks $24 \mathrm{~h}$ before use in a study. L. casei (LC) was grown in a static incubator at $37{ }^{\circ} \mathrm{C}$ in MRS media, while E. coli (EC), B. coagulans (BC), Pseudomonas aeruginosa (PA), and Salmonella typhimurium (ST) were grown in a shaking incubator $(200 \mathrm{rpm})$ at $37^{\circ} \mathrm{C}$ in Lysogeny Broth (LB) (EC, PA, ST) or Nutrient Broth (NB) (BC).

Biotinylation of Bacterial Surface: Bacteria cultures were inoculated from a glycerol stock and incubated overnight before use. Bacteria was harvested via centrifugation for $10 \mathrm{~min}$ at $4000 \mathrm{rpm}$ and washed three times with sterile, ice-cold Phosphate Buffered Saline (PBS). Biotinylation was conducted with sulfo-NHS-functionalized biotin (EZ-Link Sulfo-NHS-Biotin, ThermoFisher) with $1 \mathrm{mg}$ of sulfo-NHSbiotin per $\mathrm{mL}$ of liquid bacteria culture. All biotinylation reactions were conducted with bacteria at an OD600 of 1.0. The reaction proceeded on ice for $20 \mathrm{~min}$. Following biotinylation, bacteria were harvested via centrifugation and washed three times with ice-cold sterile PBS, as previously described. Prior to biotinylation of Visbiome surface, a single Visbiome capsule was dissolved in PBS and washed 2x in PBS to remove capsule contents.

Biotinylated Bacteria Attachment to Streptavidin: To confirm biotinylation, all biotinylated species were incubated with a 1:100 dilution of a fluorescent streptavidin conjugate (Alexa Fluor 568 Streptavidin; ThermoFisher). Bacteria were examined and imaged using an epi-fluorescence microscope (Revolve; Echo). Biotinylated EC were incubated on a streptavidin-coated plate with serial dilutions starting at $O D=0.5$. Bacteria were incubated with constant agitation $(200 \mathrm{rpm})$ for $1 \mathrm{~h}$, washed four times with sterile PBS, and fluorescence was quantified using a microplate reader (Synergy H1; BioTek).

Antibody Attachment to Biotinylated Bacteria: Antibody conjugates were formed using a commercially available streptavidin conjugation kit (Abcam) according to the manufacturer's instructions with an R6.5 anti-ICAM-1 monoclonal antibody (ThermoFisher Scientific \#BMS1011). Antibody conjugates were confirmed via protein gel electrophoresis. A total of $1 \mu \mathrm{g}$ of native protein was loaded in a TGX Stain-Free ${ }^{\text {TM Precast }}$ Gel and run according to manufacturer's instructions (Mini-PROTEAN; Bio-Rad). Bands were compared to Precision Plus Protein ${ }^{T M}$ unstained protein standards. Following confirmation of successful conjugation, antibody conjugates were incubated with biotinylated bacteria for $20 \mathrm{~min}$ with continuous agitation. Bacteria were harvested via centrifugation and washed three times as described previously to remove unbound antibody conjugates.

Bacterial Attachment to Caco-2 Cells: Caco-2 cells were seeded in tissue culture treated 96 -well plates with a cell density of $1 \times 10^{5}$ cells $\mathrm{mL}^{-1}$ and grown to confluence. Prior to the attachment study, Caco-2 cells were washed twice with pre-warmed unsupplemented media to remove FBS and pen-strep. Bacteria that were incubated with antibodies were prepared as previously described. Following the final wash, bacteria were resuspended in unsupplemented DMEM. $100 \mu \mathrm{L}$ of $3.5 \times$ $10^{9}$ cells $\mathrm{mL}^{-1}(\mathrm{OD} 600=0.5) \mathrm{EC}$ were prepared by dilution in DMEM and incubated with Caco-2 cells for $1 \mathrm{~h}$ at $37^{\circ} \mathrm{C}$. Caco-2 cells were washed four times with pre-warmed Hanks Balanced Salt Solution (HBSS) and fluorescence was quantified using a microplate reader (Synergy $\mathrm{H}$; BioTek). Unfixed cells were immediately examined and imagined under a hybrid epi-fluorescence microscope (Revolve; Echo).

Competitive Exclusion Studies: To assess bacterial toxicity toward Caco- 2 cells and the effect on the Caco-2 monolayer, cultures of EC, ST, and PA were incubated with Caco-2 monolayers for 1-h and washed $3 \mathrm{x}$ to remove unbound bacteria. Monolayer damage was assessed using microscopy on an epi-fluorescence microscope (Revolve; Echo). For exclusion studies, LC was cultured, biotinylated, and coated with ICAM-1 antibody as previously described. To confirm LC attachment to Caco-2 cells, unmodified and ICAM-1-targeted LC were incubated on a Caco-2 monolayer for $1 \mathrm{~h}$. Cells were washed to remove unbound LC, trypsinized to remove Caco-2 cells, and plated to enumerate viable, adhered LC. For the exclusion study, both LC and EC cultures were suspended in unsupplemented DMEM. Confluent Caco-2 monolayers were used for the competitive exclusion studies and were washed twice with unsupplemented DMEM prior to the study. A pilot study was conducted to determine the appropriate concentrations of EC and LC. Unmodified $L C(O D=1.0)$ was mixed with varying concentrations of $E C(O D=0.1$ to 1.0). $100 \mu \mathrm{L}$ of the bacteria mixture was incubated with Caco-2 cells for $1 \mathrm{~h}$ and washed four times with pre-warmed HBSS. Fluorescence was quantified on a plate reader, as previously described. Optimal conditions ( $E C$ at $O D=0.4, L C$ at $O D=1.0$ ) were selected, and competition studies were repeated using both unmodified LC and antibody-decorated LC.

Mouse Colonization Studies: Animal studies were conducted in accordance with and approved by the Institutional Animal Care and Use Committee (IACUC) of The University of North Carolina at Chapel Hill. 8-week old female BALB/c mice were used for in vivo colonization studies. Mice were purchased from Charles River Labs (Stock \#028) and acclimated for at least $72 \mathrm{~h}$ prior to use. Streptomycin was

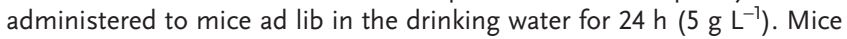
were placed back on an automatic watering system for $18 \mathrm{~h}$ prior to administration of bacteria. E. coli Nissle 1917 (EcN) with a genomically integrated GFP gene and a plasmid conferring kanamycin resistance was used for in vivo mouse colonization studies. ${ }^{[27]} \mathrm{EcN}$ was grown overnight to saturated conditions, washed to remove media, and biotinylated as described above. An anti-MUC2 antibody (Abcam \#ab76774) was conjugated to streptavidin as described above and attached to biotinylated EcN. $100 \mu \mathrm{L}$ of $10^{9} \mathrm{CFU} \mathrm{mL} \mathrm{mL}^{-1}$ bacterial culture (unmodified or anti-MUC2 modified) was administered via oral gavage to mice $(n=5$ per cage) in sterile normal saline solution for a final dose of $10^{8}$ CFU per mouse. Feces was collected starting at Day 1 by placing mice in a sterile, empty cage and waiting for approximately $2-5$ pellets of bacteria to pass. Pellets were weighed and sterile Phosphate Buffered Saline was used to homogenize the pellets. Serial dilutions of the EcN were plated on selective kanamycin $\left(50 \mu \mathrm{g} \mathrm{mL}^{-1}\right)$ plates. Colony forming units were enumerated after $72 \mathrm{~h}$ of growth at $37^{\circ} \mathrm{C}$. For studies without the use of antibiotics, $10^{9} \mathrm{CFU}$ of EcN were delivered on Day 0 via oral gavage and colonization was tracked as described.

Distribution and Abundance of $E c N$ : 8-week old female BALB/C mice were purchased from Charles River Labs (Stock \#028) and acclimated for at least $72 \mathrm{~h}$ prior to use. Streptomycin was administered to mice and unmodified and anti-MUC2-targeted EcN were prepared for oral 
gavage as described above. A bioluminescent strain of EcN was used for in vivo distribution studies to visualize the bacterial transit in the Gl tract. EcN bearing no native plasmids was transformed with pGEN-

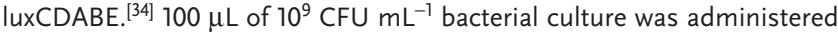
via oral gavage to mice ( $n=5$ per cage) in sterile normal saline solution. Mice were sacrificed 1- or 4-, 24-, or 72-h postgavage and the intestinal tracts were harvested, imaged with an In Vivo Imaging System (IVIS) Kinetics Optical System (PerkinElmer, CA), and segmented into the small intestine, cecum and colon. Fecal samples were collected from mice in the 24- and 72-h cohorts at 6-, 12-, 18-, 24-, 48-, and $72-h$ postgavage and processed as described above. All intestinal segments were homogenized twice with an MP Biomedical FastPrep-24 homogenizer using $1.4 \mathrm{~mm}$ ceramic bead-filled tubes (15 seconds, $6.5 \mathrm{M} \mathrm{s}^{-1}$ ). Intestinal samples were serially diluted and plated on ampicillin (100 $\left.\mu \mathrm{g} \mathrm{mL} \mathrm{m}^{-1}\right)$ selective LB agar plates. Viable colony forming units were enumerated and data was normalized to the dose given to each animal. All IVIS images were scaled to visualize the lowest signal in each image.

\section{Supporting Information}

Supporting Information is available from the Wiley Online Library or from the author.

\section{Acknowledgements}

A.M.V. was supported by the National Science Foundation Graduate Research Fellowship under Grant No. DGE-1650116. This study was financially supported in part from the PharmAlliance, a three-way partnership between the schools of pharmacy at the University of North Carolina at Chapel Hill, the University College London, and Monash University.

\section{Conflict of Interest}

A.M.V. and A.C.A. are inventors on a provisional patent application filed by The University of North Carolina at Chapel Hill on the work presented here.

\section{Keywords}

biomaterials, drug delivery, microbiome, probiotics, targeted delivery

Received: March 15, 2020

Revised: April 8, 2020

Published online: May 14, 2020

[1] a) J. R. Brestoff, D. Artis, Nat. Immunol. 2013, 14, 676; b) C. G. Buffie, E. G. Pamer, Nat. Rev. Immunol. 2013, 13, 790; c) C. G. Buffie, V. Bucci, R. R. Stein, P. T. McKenney, L. Ling, A. Gobourne, D. No, H. Liu, M. Kinnebrew, A. Viale, E. Littmann, M. R. van den Brink, R. R. Jenq, Y. Taur, C. Sander, J. R. Cross, N. C. Toussaint, J. B. Xavier, E. G. Pamer, Nature 2015, 517, 205.

[2] A. M. Vargason, A. C. Anselmo, Bioeng. Transl. Med. 2018, 3, 124.

[3] a) J. S. Bakken, T. Borody, L. J. Brandt, J. V. Brill, D. C. Demarco, M. A. Franzos, C. Kelly, A. Khoruts, T. Louie, L. P. Martinelli, T. A. Moore, G. Russell, C. Surawicz, Clin. Gastroenterol. Hepatol. 2011, 9, 1044; b) K. F. Blount, W. D. Shannon, E. Deych, C. Jones, Open Forum Infect. Dis. 2019, 6, 095; c) C. Staley, M. J. Hamilton,
B. P. Vaughn, C. T. Graiziger, K. M. Newman, A. J. Kabage, M. J. Sadowsky, A. Khoruts, Am. J. Gastroenterol. 2017, 112, 940.

[4] V. Jacob, C. Crawford, S. Cohen-Mekelburg, M. Viladomiu, G. G. Putzel, Y. Schneider, F. Chabouni, S. O'Neil, B. Bosworth, V. Woo, N. J. Ajami, J. F. Petrosino, Y. Gerardin, Z. Kassam, M. Smith, I. D. Iliev, G. F. Sonnenberg, D. Artis, E. Scherl, R. S. Longman, Inflammatory Bowel Dis. 2017, 23, 903.

[5] V. Gopalakrishnan, B. A. Helmink, C. N. Spencer, A. Reuben, J. A. Wargo, Cancer Cell 2018, 33, 570.

[6] V. M. Isabella, B. N. Ha, M. J. Castillo, D. J. Lubkowicz, S. E. Rowe, Y. A. Millet, C. L. Anderson, N. Li, A. B. Fisher, K. A. West, P. J. Reeder, M. M. Momin, C. G. Bergeron, S. E. Guilmain, P. F. Miller, C. B. Kurtz, D. Falb, Nat. Biotechnol. 2018, 36, 857.

[7] C. B. Kurtz, Y. A. Millet, M. K. Puurunen, M. Perreault, M. R. Charbonneau, V. M. Isabella, J. W. Kotula, E. Antipov, Y. Dagon, W. S. Denney, D. A. Wagner, K. A. West, A. J. Degar, A. M. Brennan, P. F. Miller, Sci. Transl. Med. 2019, 11, 7975.

[8] a) J. Walter, M. X. Maldonado-Gomez, I. Martinez, Curr. Opin. Biotechnol. 2018, 49, 129; b) C. S. Smillie, J. Sauk, D. Gevers, J. Friedman, J. Sung, I. Youngster, E. L. Hohmann, C. Staley, A. Khoruts, M. J. Sadowsky, J. R. Allegretti, M. B. Smith, R. J. Xavier, E. J. Alm, Cell Host Microbe 2018, 23, 229.

[9] a) M. Mimee, R. J. Citorik, T. K. Lu, Adv. Drug Delivery Rev. 2016, 105, 44; b) D. Kao, B. Roach, M. Silva, P. Beck, K. Rioux, G. G. Kaplan, H. J. Chang, S. Coward, K. J. Goodman, H. Xu, K. Madsen, A. Mason, G. K. Wong, J. Jovel, J. Patterson, T. Louie, JAMA, J. Am. Med. Assoc. 2017, 318, 1985; c) T. H. Joeres-Nguyen-Xuan, S. K. Boehm, L. Joeres, J. Schulze, W. Kruis, Inflammatory Bowel Dis. 2010, 16, 256.

[10] G. P. Donaldson, S. M. Lee, S. K. Mazmanian, Nat. Rev. Microbiol. 2016, 14, 20.

[11] a) T. D. Lawley, A. W. Walker, Immunology 2013, 138, 1; b) M. Sassone-Corsi, M. Raffatellu, J. Immunol. 2015, 194, 4081.

[12] a) A. C. Anselmo, K. J. McHugh, J. Webster, R. Langer, A. Jaklenec, Adv. Mater. 2016, 28, 9486; b) Z. Cao, X. Wang, Y. Pang, S. Cheng, J. Liu, Nat. Commun. 2019, 10, 5783; c) M. T. Cook, G. Tzortzis, D. Charalampopoulos, V. V. Khutoryanskiy, J. Controlled Release 2012, 162, 56; d) Z. Li, A. M. Behrens, N. Ginat, S. Y. Tzeng, X. Lu, S. Sivan, R. Langer, A. Jaklenec, Adv. Mater. 2018, 30, 1803925.

[13] a) Y. H. Bae, K. Park, J. Controlled Release 2011, 153, 198; b) R. Singh, J. W. Lillardjr., Exp. Mol. Pathol. 2009, 86, 215; c) S. C. Alley, N. M. Okeley, P. D. Senter, Curr. Opin. Chem. Biol. 2010, 14, 529.

[14] a) W. Chen, Y. Wang, M. Qin, X. Zhang, Z. Zhang, X. Sun, Z. Gu, ACS Nano 2018, 12, 5995; b) S. Patyar, R. Joshi, D. P. Byrav, A. Prakash, B. Medhi, B. Das, J. Biomed. Sci. 2010, 17, 21; c) C. M. Cheng, Y. L. Lu, K. H. Chuang, W. C. Hung, J. Shiea, Y. C. Su, C. H. Kao, B. M. Chen, S. Roffler, T. L. Cheng, Cancer Gene Ther. 2008, 15, 393; d) Z. Cao, S. Cheng, X. Wang, Y. Pang, J. Liu, Nat. Commun. 2019, 10, 3452.

[15] a) C. Pinero-Lambea, G. Bodelon, R. Fernandez-Perianez, A. M. Cuesta, L. Alvarez-Vallina, L. A. Fernandez, ACS Synth. Biol. 2015, 4, 463; b) F. Al-Saedi, D. H. Stones, D. P. Vaz, A. M. Krachler, Infect. Immun. 2016, 84, 1704; c) D. T. Riglar, P. A. Silver, Nat. Rev. Microbiol. 2018, 16, 214.

[16] a) H. P. Lesch, M. U. Kaikkonen, J. T. Pikkarainen, S. Ylä-Herttuala, Expert Opin. Drug Delivery 2010, 7, 551; b) J. N. Hathcock, J. C. Griffiths, Vitamin and Mineral Safety, 3rd ed.Council for Responsible Nutrition, xxxx 2014.

[17] M. Hartmann, M. Berditsch, J. Hawecker, M. F. Ardakani, D. Gerthsen, A. S. Ulrich, Antimicrob. Agents Chemother. 2010, 54, 3132.

[18] M. Lizier, P. G. Sarra, R. Cauda, F. Lucchini, FEMS Microbiol. Lett. 2010, 308, 8 . 
[19] D. Kaiserlian, D. Rigal, J. Abello, J. P. Revillard, Eur. J. Immunol. 1991, 21, 2415.

[20] W. W. Wilson, M. M. Wade, S. C. Holman, F. R. Champlin, J. Microbiol. Methods 2001, 43, 153.

[21] J. D. Ramakers, R. P. Mensink, G. Schaart, J. Plat, Lipids 2007, 42, 687.

[22] T. R. Callaway, T. S. Edrington, R. C. Anderson, R. B. Harvey, K. J. Genovese, C. N. Kennedy, D. W. Venn, D. J. Nisbet, Anim. Health Res. Rev. 2008, 9, 217.

[23] a) A. L. Servin, FEMS Microbiol. Rev. 2004, 28, 405; b) M. F. Bernet, D. Brassart, J. R. Neeser, A. L. Servin, Gut 1994, 35, 483.

[24] a) S. Matsumoto, T. Hara, T. Hori, K. Mitsuyama, M. Nagaoka, N. Tomiyasu, A. Suzuki, M. Sata, Clin. Exp. Neuroimmunol. 2005, 140, 417; b) M. Gueimonde, L. Jalonen, F. He, M. Hiramatsu, S. Salminen, Food Res. Int. 2006, 39, 467.

[25] a) M. Hussain, D. Schäfer, K. M. Juuti, G. Peters, B. HaslingerLöffler, P. I. Kuusela, B. Sinha, J. Infect. Dis. 2009, 200, 107; b) Y. K. Lee, K. Y. Puong, Br. J. Nutr. 2002, 88, 101.

[26] a) W. Kruis, P. Fric, J. Pokrotnieks, M. Lukas, B. Fixa, M. Kascak, M. A. Kamm, J. Weismueller, C. Beglinger, M. Stolte, C. Wolff, J. Schulze, Gut 2004, 53, 1617; b) S. N. Ukena, A. Singh, U. Dringenberg, R. Engelhardt, U. Seidler, W. Hansen, A. Bleich, D. Bruder, A. Franzke, G. Rogler, S. Suerbaum, J. Buer, F. Gunzer, A. M. Westendorf, PLoS One 2007, 2, 1308; c) M. Schultz, Inflammatory Bowel Dis. 2008, 14, 1012; d) J. Henker, M. Laass, B. M. Blokhin, Y. K. Bolbot, V. G. Maydannik, M. Elze, C. Wolff, J. Schulze, Eur. J. Pediatr. 2007, 166, 311.

[27] N. Crook, A. Ferreiro, A. J. Gasparrini, M. W. Pesesky, M. K. Gibson, B. Wang, X. Sun, Z. Condiotte, S. Dobrowolski, D. Peterson, G. Dantas, Cell Host Microbe 2019, 25, 499.

[28] a) M. E. Johansson, J. M. Larsson, G. C. Hansson, Proc. Natl. Acad. Sci. USA 2011, 108, 4659; b) J. F. Sicard, G. Le Bihan, P. Vogeleer, M. Jacques, J. Harel, Front. Cell. Infect. Microbiol. 2017, 7, 387.

[29] a) M. J. Hamilton, A. R. Weingarden, M. J. Sadowsky, A. Khoruts, Am. J. Gastroenterol. 2012, 107, 761; b) S. Khanna, D. S. Pardi,
C. R. Kelly, C. S. Kraft, T. Dhere, M. R. Henn, M. J. Lombardo, M. Vulic, T. Ohsumi, J. Winkler, C. Pindar, B. H. McGovern, R. J. Pomerantz, J. G. Aunins, D. N. Cook, E. L. Hohmann, J. Infect. Dis. 2016, 214, 173.

[30] a) M. Bohnhoff, B. L. Drake, C. P. Miller, Exp. Biol. Med. 1954, 86, 132; b) D. J. Hentges, P. Pongpech, J. U. Que, Microb. Ecol. Health Dis. 1990, 3, 105; c) C. Tyrrell, P. S. Cohen, in Virulence Mechanisms of Bacterial Pathogens, 4th ed., American Society of Microbiology, xxxx 2007.

[31] a) E. S. Lamouse-Smith, A. Tzeng, M. N. Starnbach, PLoS One 2011, 6, 27662; b) P. Praveschotinunt, A. M. Duraj-Thatte, I. Gelfat, F. Bahl, D. B. Chou, N. S. Joshi, Nat. Commun. 2019, 10, 5580; c) A. L. Goodman, G. Kallstrom, J. J. Faith, A. Reyes, A. Moore, G. Dantas, J. I. Gordon, Proc. Natl. Acad. Sci. USA 2011, 108, 6252.

[32] C. Kurtz, W. S. Denney, L. Blankstein, S. E. Guilmain, S. Machinani, J. Kotula, S. Saha, P. Miller, A. M. Brennan, Clin. Transl. Sci. 2018, 11, 200.

[33] a) Z. Matuskova, E. Anzenbacherova, R. Vecera, H. TlaskalovaHogenova, M. Kolar, P. Anzenbacher, PLoS One 2014, 9, 87150; b) N. Pavlović, K. Stankov, M. Mikov, Appl. Biochem. Biotechnol. 2012, 168, 1880; c) J.-K. Kim, M. S. Choi, J.-J. Jeong, S.-M. Lim, I. S. Kim, H. H. Yoo, D.-H. Kim, Drug Metab. Dispos. 2018, 46, 122; d) P. Marteau, T. Tuure, Biosci. Microflora 1998, 17, 1; e) G. Blandino, D. Fazio, R. Di Marco, Expert Rev. Anti-Infect. Ther. 2008, 6, 497.

[34] M. C. Lane, C. J. Alteri, S. N. Smith, H. L. Mobley, Proc. Natl. Acad. Sci. USA 2007, 104, 16669.

[35] L. H. Lam, D. M. Monack, PLoS Pathog. 2014, 10, 1004527.

[36] V. M. Isabella, B. N. Ha, M. J. Castillo, D. J. Lubkowicz, S. E. Rowe, Y. A. Millet, C. L. Anderson, N. Li, A. B. Fisher, K. A. West, P. J. Reeder, M. M. Momin, C. G. Bergeron, S. E. Guilmain, P. F. Miller, C. B. Kurtz, D. Falb, Nat. Biotechnol. 2018, 36, 857.

[37] A. C. Dukowicz, B. E. Lacy, G. M. Levine, Gastroenterol. Hepatol. 2007, 3, 112 . 